\title{
Effects of maximum strain and aging time on the fatigue lifetime of vulcanized natural rubber
}

\author{
C. S. Woo \& H. S. Park \\ Department of Nano Mechanics Team, \\ Korea Institute of Machinery and Materials, Korea
}

\begin{abstract}
Fatigue life prediction and evaluation are the key technologies to assure the safety and reliability of automotive rubber components. In this study, a fatigue lifetime prediction equation was derived to exactly estimate the fatigue lifetimes of anti-vibration rubber components in a short period at the initial stage of design. A fatigue lifetime prediction methodology of vulcanized natural rubber was proposed by incorporating the finite element analysis and fatigue damage parameter determined from the fatigue test. Nonlinear finite element analyses of the 3-dimensional dumbbell specimen and rubber component were performed using an Ogden hyper-elastic material model determined from the tensile, shear and biaxial tension tests of the natural rubber, and resulted in relationships between displacement and the Green-Lagrange strain for both the dumbbell specimen and rubber component. Fatigue tests were performed using a 3dimensional dumbbell specimen at different levels of maximum displacement and heat aging temperature. It was shown that the maximum tension displacement was a proper damage parameter, taking the mean displacement effect into account. A procedure to predict the fatigue lifetime of vulcanized natural rubber material based on the maximum strain method was proposed. With the proposed fatigue lifetime equation, the fatigue lifetime could be exactly estimated within the error range of $200 \%$, irrespective of rubber material and aging time.

Keywords: rubber component, fatigue test, fatigue lifetime prediction, finite element analysis, maximum Green-Lagrange strain.
\end{abstract}




\section{Introduction}

Rubber's ability to withstand great strain without permanent deformation or fracture makes it ideal for many applications. Applications include vibration isolators, seals, structural bearing, to name a few. These applications impose large static and time-varying strains over a long time. Long-time durability is therefore a critical issue. While many factors contribute to long-time durability, mechanical fatigue, the nucleation and growth of cracks in the rubber, is often the primary consideration [1]. To address the issue effectively and economically, engineers need to model and design for mechanical fatigue early in the product development process. Most rubber components are subjected to static and dynamic loadings in service. Preventing failures during operation is one of the critical issues in rubber component design [2]. Therefore, fatigue analysis and lifetime evaluation are very important in the design procedure to assure the safety and reliability of the mechanical rubber component [3, 4]. In order to achieve an excellent result of fatigue lifetime and have short time test cycles, we expected the development of a new method of fatigue test. As result, we have researched to find a new fatigue test, which is able to anticipate the fatigue life of a rubber component, by the use of correlations with actual vehicle parts by a conventional fatigue test, at the stage of the material development test. This paper shows a fatigue test using a newly designed test piece, which is supposed to simulate actual parts strain by the finite element analysis method, and also shows the relationship between the fatigue life of the test piece and the actual parts, using the FEM analyzed strain as a parameter. In this study, by using the parameter of maximum Green-Lagrange strains appearing at the locations, we can prove the relations between fatigue life and maximum Greenge appear strain, and the correlations between the test piece test and the bench test of the actual rubber component. In order to predict the fatigue life of rubber components at the design stage, a simple procedure of lifetime prediction is suggested in Fig. 1.

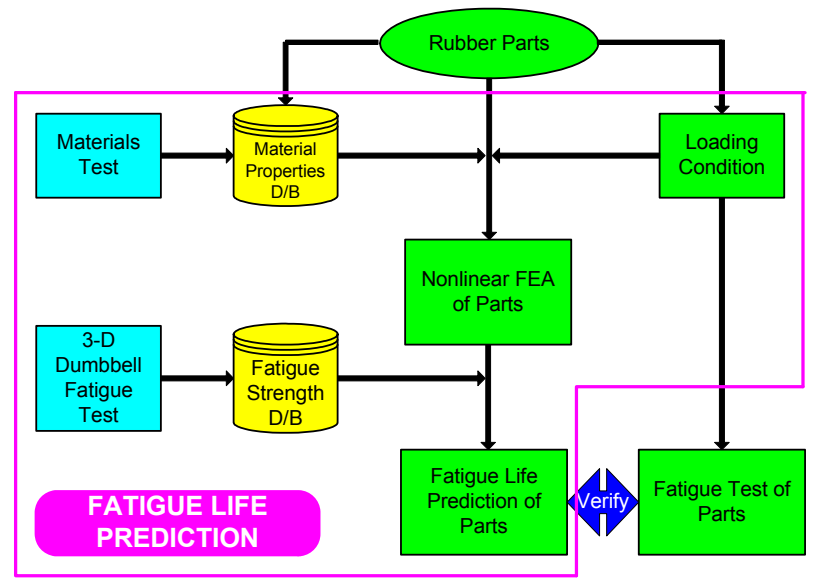

Figure 1: Procedure of the fatigue lifetime prediction system for a rubber component. 


\section{Experiments}

\subsection{Rubber materials}

The material used in this study is carbon-filled vulcanized natural rubber, which has the hardness of the International Rubber Hardness Degree 50, 55, 60, 65 (NR50, NR55, NR60, NR65). Compound recipes, including applied cure conditions, are summarized in Table 1.

Table 1: Compound recipes investigated of natural rubber.

\begin{tabular}{|c|c|c|c|c|}
\hline Ingredient & NR50 & NR55 & NR60 & NR65 \\
\hline SMRCV 60 & 100 & 100 & 100 & 100 \\
\hline C/B FEF & 22 & 27 & 40 & 40 \\
\hline C/B SRF & 15 & 18 & 20 & 32 \\
\hline S/A & 1 & 1 & 1 & 1 \\
\hline ZnO & 5 & 5 & 5 & 5 \\
\hline
\end{tabular}

\subsection{Fatigue test specimen}

The test piece has the basic shape of the three-dimensional dumbbell specimen with a metal fitting cure bonded to each end. The geometry of the central part of the cylinder was designed to meet the following criteria in relation to fatigue test data for rubber components and a strain distribution profile. The test piece should be capable of compression and tensile deformation without developing slackness under cyclic deformation. It should have a smooth strain distribution and the position at which maximum tensile strain develops should be the same for any deformation. A three-dimensional dumbbell specimen has an elliptical cross-section and parting lines are located on the minor axis of the specimen to avoid undesirable failure at the surface discontinuities [5]. The basic geometry of the test piece for material fatigue testing is shown in Fig. 2(a). The finite element analysis is executed to obtain the relationship between the displacement and strain [6]. It was assumed that the ends of the specimen were constrained by bonding to the fitting and the rubber part was deformed in the longitudinal direction. A finite element model of the specimen is shown in Fig. 2(b). The maximum strain was found in the surface at the major axis of the rubber part of the specimen (as shown in Fig. 2(c)). To avoid the mold parting line acting as a source of crack initiation, the parting line should be placed on the minor axis side. Fig. 2(d) shows the strain distribution according to finite element analysis from compression and tension displacement. The maximum Green-Lagrange strain was found to develop at a constant position on the surface at the centre of the rubber part of the test piece in both compression and tension. 


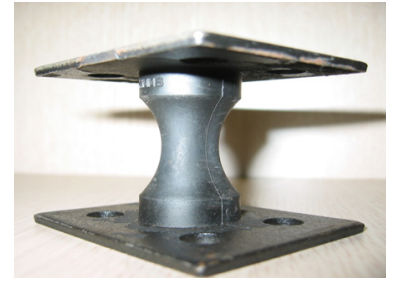

(a)

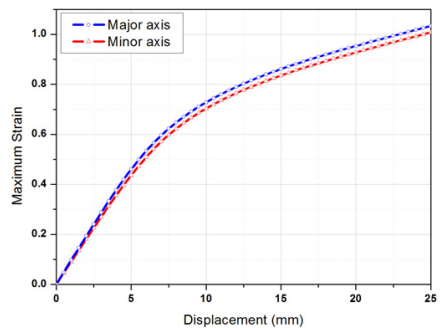

(c)

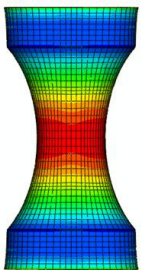

(b)

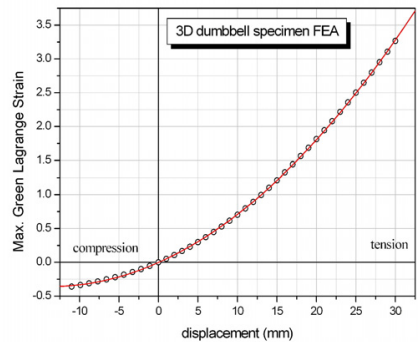

(d)

Figure 2: Three-dimensional dumbbell specimen for fatigue test: (a) threedimensional dumbbell specimen; (b) finite element model; (c) strain distribution of specimen; (d) displacement and strain curve.

\subsection{Fatigue test}

In order to evaluate a fatigue damage parameter of the natural rubber material and to determine the experimental fatigue life, fatigue tests of a threedimensional dumbbell specimen were performed using the fatigue testing system. Fatigue tests were conducted in an ambient and heat aging (90 days at $70^{\circ} \mathrm{C}$ ) temperature under the stroke-controlled condition with a sine waveform of $5 \mathrm{~Hz}$ and the mean displacement is $0,3,5,8,10 \mathrm{~mm}$ at the displacement range is -11$21 \mathrm{~mm}$. Fatigue failure was defined as the number of cycles at which the maximum load dropped by 20 percent. With increasing the cycles in the initial phase, the maximum load decreased little by little. When the crack grew over the critical size, the maximum load decreased suddenly and the final failure was reached. Fig. 3 shows the fatigue testing system and fatigue life curve.

\section{Result and discussion}

\subsection{Fatigue damage parameter}

Fig. 4 shows the relationship between displacement and fatigue life for the threedimensional dumbbell specimens with different hardnesses under various mean displacements. The fatigue life at the same displacement amplitude decreases as the mean displacement and hardness increases. 


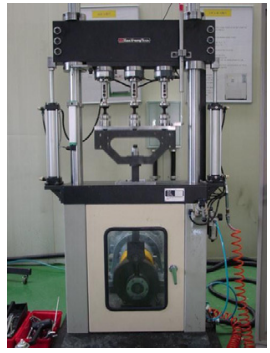

(a)

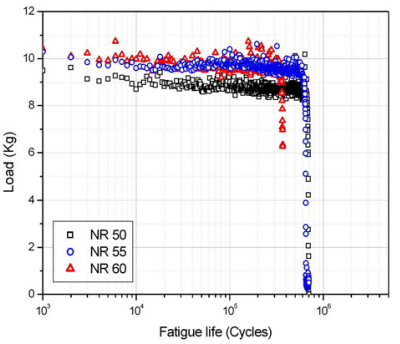

(b)

Figure 3: Fatigue test of a three-dimensional dumbbell specimen: (a) fatigue testing system; (b) definition of fatigue failure.

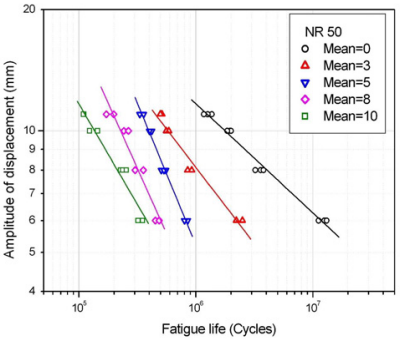

(a)

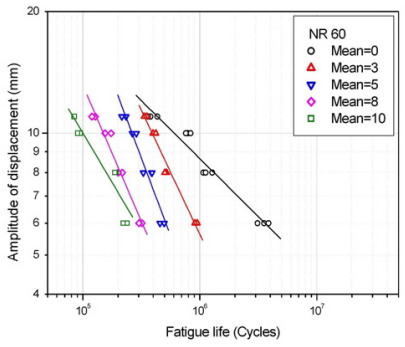

(c)

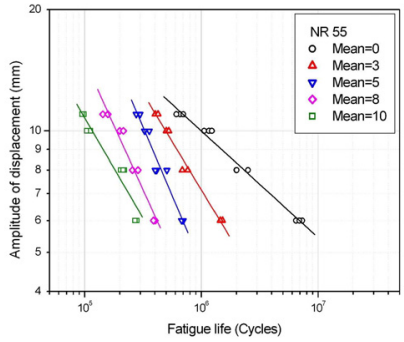

(b)

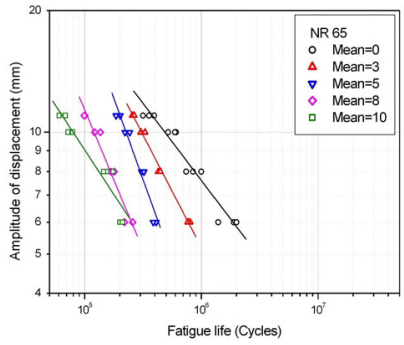

(d)

Figure 4: Fatigue life of a three-dimensional dumbbell specimen: (a) NR 50; (b) NR 55; (c) NR 60; (d) NR 65.

The fatigue process begins with the accumulation of damage at a localized region due to alternating load and displacement, leading to crack nucleation, growth, and final fracture $[7,8]$. The crack nucleation life of the component may be defined as the number of cycles required for the appearance of a macro-crack. Therefore, the crack nucleation life of the component can be related to the life of a smooth specimen that is cycled to the same stresses or strains as the material at the critical region of the component. In this study, the fatigue damage of the natural rubber was evaluated from three-dimensional dumbbell specimens. Fig. 5(a) shows the relationship between the maximum tension displacement and 
fatigue life. The fatigue life decreases as the maximum tension displacement increases. It is possible to express the fatigue life with maximum tension displacement as fairly good. The relationship between the applied displacement and the corresponding Green-Lagrange strain of the three-dimensional dumbbell specimen are obtained from finite element analysis and is shown in Fig. 2. The fatigue life curve of natural rubber expressed by the Green-Lagrange strain as a damage parameter can be generated from the displacement versus the GreenLagrange strain curve of the three-dimensional specimen. Fig. 5(b) shows the maximum Green-Lagrange strain versus cycles to failure of natural rubber.

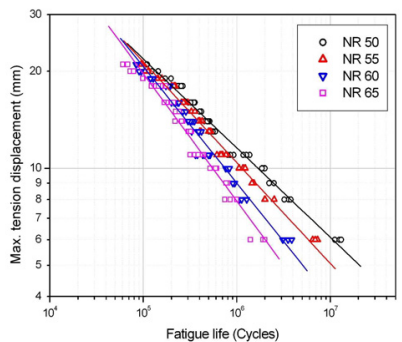

(a)

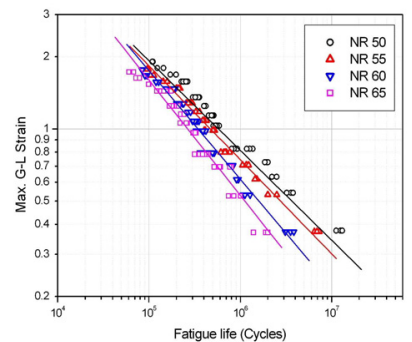

(b)

Figure 5: Maximum displacement and $\mathrm{G}-\mathrm{L}$ strain versus cycles to failure: (a) maximum displacement; (b) maximum G-L strain.

\subsection{Fatigue lifetime prediction}

Fatigue life of the three-dimensional dumbbell specimen represented by the maximum Green-Lagrange strain parameter $\left(\varepsilon_{\mathrm{G}-\mathrm{L}}\right)$ is shown in Fig. $5(\mathrm{~b})$, where the Green-Lagrange strain for each dumbbell specimen is calculated from the displacement versus Green-Lagrange strain curve in Fig. 5(a). It can be seen from Fig. 5(b) that the fatigue life with different hardnesses can be effectively represented by the following function using the maximum Green-Lagrange strain, thus taking into account the mean displacement and amplitude. The Green-Lagrange strain was defined as equation (1).

$$
\varepsilon_{G-L}=\frac{1}{2}\left(\lambda^{2}-1\right)
$$

$\varepsilon_{G-L}:$ Green-Lagrange strain, $\lambda:$ stretch ratio.

By using the fatigue test and finite element analysis, the normalized maximum strain is defined as dividing by elongation at break $\left(\varepsilon_{\mathrm{EB}}\right)$ for the maximum Green-Lagrange strain $\left(\varepsilon_{\mathrm{G}-\mathrm{L}}\right)$ for each hardness. Fig. 6 shows the relation of normalized maximum strain and fatigue life. It was observed that the maximum G-L strain was a good parameter to account for the hardness, mean displacement and amplitude effects. The fatigue life of the threedimensional dumbbell specimen represented by the maximum $\mathrm{G}-\mathrm{L}$ strain and 
elongation at break are shown in equation (2). Fatigue life is effectively represented by a single function using the maximum G-L strain and elongation at break for each natural rubber material.

$$
N_{f}=1,096 \cdot\left[\varepsilon_{G-L} / \varepsilon_{E B}\right]^{-2.22}
$$

Fig. 6(c) compares the predicted and experimental fatigue lives for the variable amplitude signals for natural rubber in terms of sequences to failure along with reference lines indicating factors of 2 scatter in fatigue life.

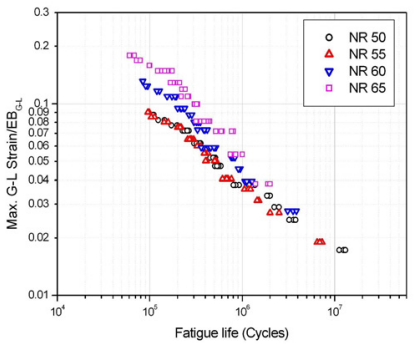

(a)

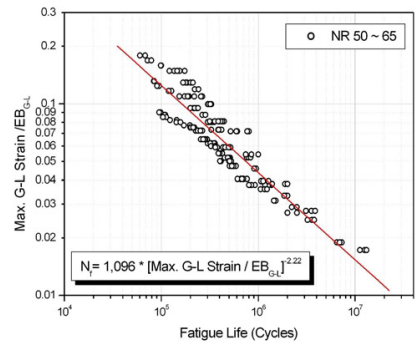

(b)

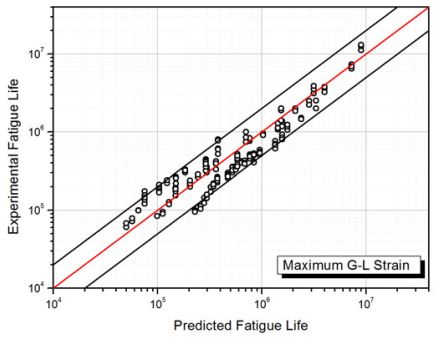

(c)

Figure 6: Fatigue lifetime prediction curve of natural rubber: (a) maximum G-L strain versus fatigue life; (b) fatigue life prediction curve; (c) predicted and experimental life.

\subsection{Fatigue lifetime in heat aging conditions}

The estimation of fatigue lifetime of aged rubber specimens should use the results of the aging characteristics tests and aging fatigue lifetime tests. Because the fatigue tests were conducted while rubber specimens of each material were aged in an environmental chamber at $70^{\circ} \mathrm{C}$, the aging characteristics of aged specimens at $70^{\circ} \mathrm{C}$ for 90 days should be used for the mechanical properties of the material. Because fatigue lifetime damage variables were elongation and maximum strain when the rubber was broken as well as the maximum displacement of rubber when the fatigue lifetime was estimated at heat aging temperature, these variables were used to estimate aging fatigue lifetime. As 
shown in Fig. 7(a), elongation decreased as aging times were long. The relation of the maximum $\mathrm{G}-\mathrm{L}$ strain and aging time obtained by finite element analysis was relatively constant irrespective of aging time (as shown in Fig. 7(b)). Using the relation of the maximum $\mathrm{G}-\mathrm{L}$ strain and aging time, the relation of the maximum tensile displacement and fatigue lifetime obtained in aging fatigue tests was represented by the relation of the maximum G-L strain and fatigue lifetime as shown in Fig. 8. In this figure, fatigue lifetime decreased as aging time increased, and it significantly changed depending on aging time and materials.

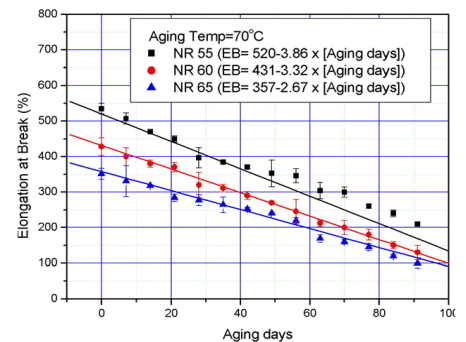

(a)

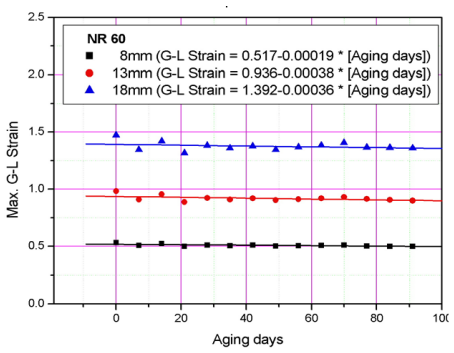

(b)

Figure 7: Effect of aging on elongation and maximum $\mathrm{G}-\mathrm{L}$ strain: (a) elongation at break; (b) maximum G-L strain.

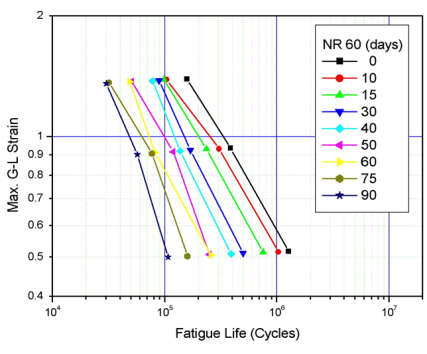

(a)

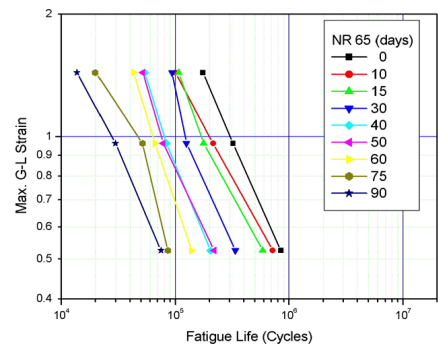

(b)

Figure 8: Maximum G-L strain vs. fatigue life for aged specimens: (a) NR 60; (b) NR 65 .

The fatigue lifetime of aged specimens could be estimated using the same method as the estimation of fatigue lifetime at ambient temperatures. The dimensionless variable was obtained (as shown in Fig. 9), by dividing the maximum $\mathrm{G}-\mathrm{L}$ strain by elongation according to aging time, irrespective of rubber materials and aging time. And then the following equation to estimate fatigue lifetime was obtained in Eq. (3): 


$$
N_{f}=3,846 \cdot\left[\epsilon_{G-L} / \epsilon_{E B}\right]^{-1.757}
$$

Fig. 9(d) shows the relation of fatigue lifetime estimated by Eq. (3) and through the experiments. It was found that they were well consistent within the error range of $200 \%$. Using the fatigue damage variable that was obtained by dividing the maximum $\mathrm{G}-\mathrm{L}$ strain by elongation when the rubber was broken, fatigue lifetime was well estimated irrespective of rubber materials and aging time.

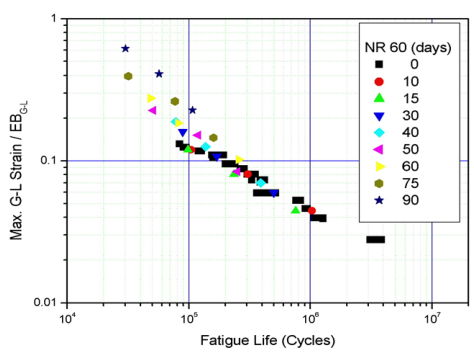

(a)

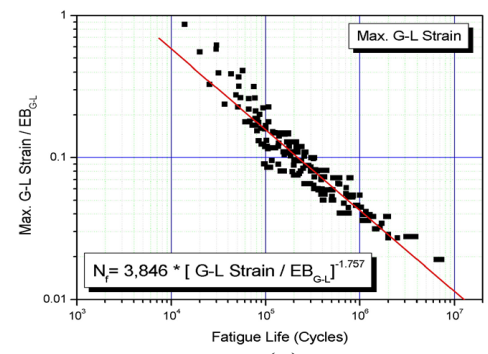

(c)

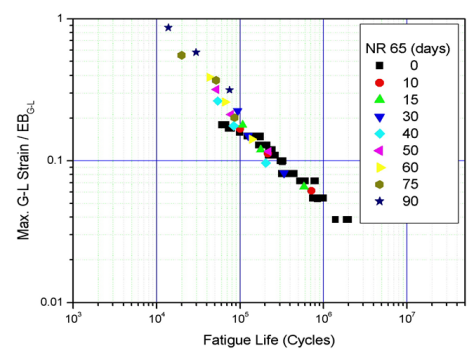

(b)

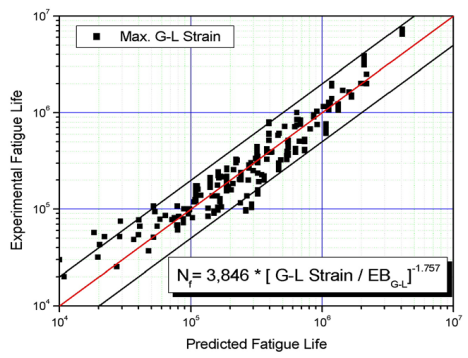

(d)

Figure 9: Fatigue lifetime prediction curve of heat aged rubber material: (a) NR 60; (b) NR 65; (c) fatigue life prediction curve; (d) predicted and experimental life.

\subsection{Fatigue lifetime evaluation of rubber component}

The methodology was applied to the fatigue life estimation of a rubber engine mount. Finite element analysis was performed to investigate the deformation behaviour of the rubber component. Material constants representing the Ogden strain energy potential of order 3 was used for defining a constitutive relation of the natural rubber. Fig. 10 shows the Green-Lagrange strain distribution of a rubber engine mount under tensile displacement. The maximum Green-Lagrange strain at the critical location was used for evaluating the fatigue damage parameter of a rubber engine mount. Static and fatigue tests were performed using a servo-hydraulic fatigue testing system shown in Fig. 11. The loaddisplacement curve is very similar to the correlation between the test and FEM. 
Maximum Green-Lagrange strain occurred at the location as indicated in Fig. 12, and fatigue cracking at the critical location was observed during the fatigue test of the rubber engine mount. The correlation between the fatigue test life and predicted life of the rubber engine mount using the normalized maximum strain is shown in Fig. 13. The predicted fatigue lives of the rubber engine mount agreed fairly well with the experimental fatigue lives.

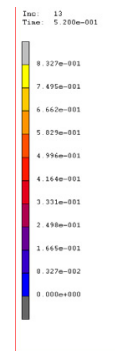

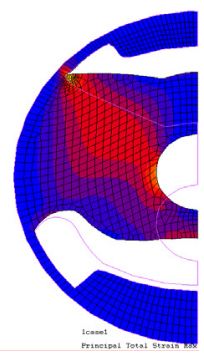

(a)

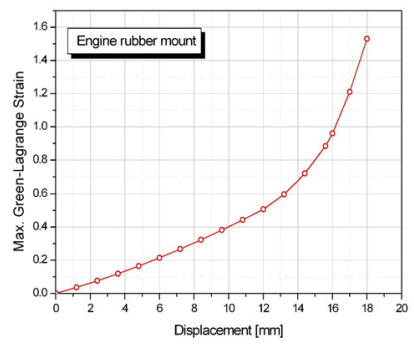

(b)

Figure 10: Green-Lagrange strain distribution of engine rubber mount: (a) finite element analysis; (b) maximum G-L strain versus displacement.

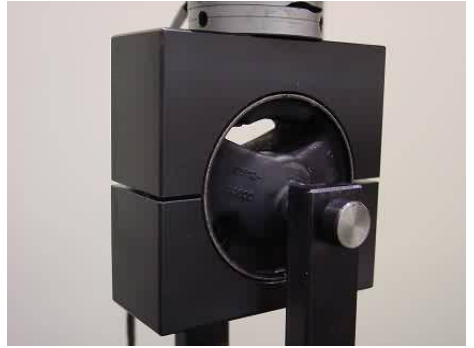

(a)

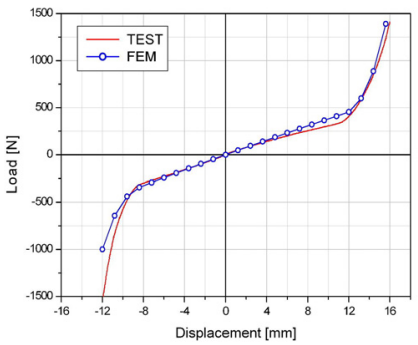

(b)

Figure 11: Static test and correlation between test and FEM: (a) static test; (b) correlation between test and FEM.

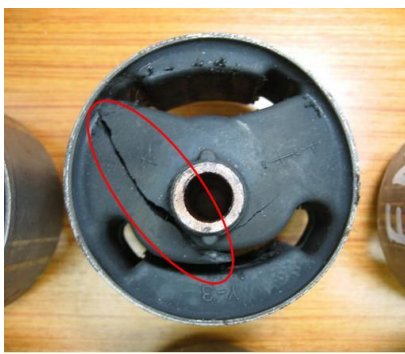

(a)

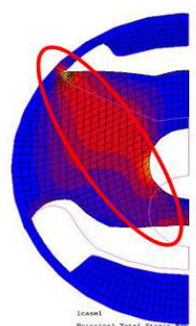

(b)

Figure 12: Fatigue test and failure of engine rubber mount: (a) fatigue failure of engine mount; (b) distribution of G-L strain. 


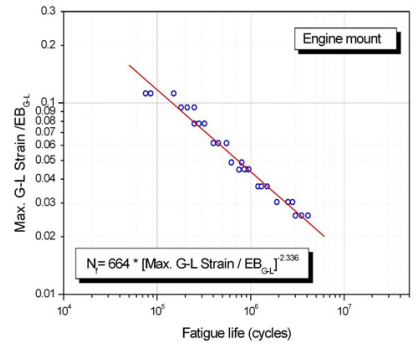

(a)

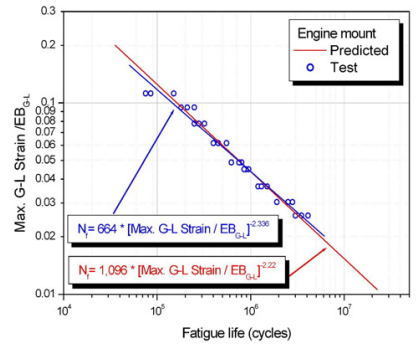

(b)

Figure 13: Evaluation of fatigue life for rubber engine mount: (a) fatigue life of engine mount; (b) compare with predicted and test.

\section{Conclusion}

Fatigue lifetime evaluation of vulcanized rubber material was very important in the design procedure to assure the safety and reliability of the rubber components. In this paper, fatigue lifetime prediction methodology of the rubber components was proposed by incorporating the finite element analysis and fatigue damage parameter determined from fatigue tests. The fatigue lifetime of a rubber component was effectively represented by the maximum Green-Lagrange strain. The predicted fatigue lifetime of the rubber component was in fairly good agreement with the experimental lifetime. Therefore, the fatigue lifetime estimation procedure employed in this study could be used approximately for the fatigue design of rubber components at the early design stage.

\section{Acknowledgement}

This research has been supported by Development of Materials and Component Program of Ministry Commerce Industry and Energy, Korea.

\section{References}

[1] Gent, A.N, Strength of Elastomer, Science and Technology of Rubber, Mark JE, $2^{\text {nd }}$ ed., Academic Press, Chapter No. 10, pp. 471-512, 1994.

[2] Lake, G.J, Fatigue and Fracture of Elastomers, Rubber Chem. \& Technology, vol. 68, pp. 435-460, 1995.

[3] Lake, Aspect of Fatigue and Fracture of Rubber, Progress of rubber tech., vol. 45, pp. 89-143, 1983.

[4] Klenke, D., Beste, A., Ensurance of the fatigue life of metal rubber component, Gummi Kunsftoffe, vol. 40, pp. 1067-1071, 1987.

[5] Yamaguchi, H. \& Nakagawa M., Fatigue Test Technique for Rubber materials of Vibration Insulator, International Polymer Science and Technology, vol. 20, pp. 64-69, 1993. 
[6] Lee, S., Yeom, S., Han, C., Woo, C., A study on finite element analysis and aging test for automotive grommer, Elastomer \& Composites, vol. 47, pp. 201-209, 2012.

[7] Oh, H.L, The fatigue life model of rubber bushing, Rubber Chem. \& Technology, vol. 53, pp. 1226-1238, 1980.

[8] Alshuth, T. \& Abraham F., Parameter Dependence and Prediction of Fatigue life of Elastomers products, Rubber Chem. \& Technology, vol. 75 pp. 635642, 2002. 